\title{
RESEARCH
}

Open Access

\section{Evaluation of a novel ovarian cancer- specific fluorescent antibody probe for targeted near-infrared fluorescence imaging}

Junchen Chen ${ }^{1,2}$, Chen Zhang ${ }^{1,2}$, Yanxiu Guo ${ }^{1,2}$, Xiaohong Chang ${ }^{1,2}$, Ruiqiong Ma ${ }^{1,2}$, Xue Ye $e^{1,2}$, Hongyan Cheng ${ }^{1,2}$, Yi Li ${ }^{1 *}$ and Heng Cui ${ }^{1,2^{*}}$

\begin{abstract}
Background: To meet clinical needs, fluorescence-guided surgery has emerged as a new technique that guides surgeons in the resection of cancerous tissue by highlighting tumour lesions during surgery. We aimed to evaluate the novel ovarian cancer-specific antibody fluorescent probe COC183B2-800 (COC183B2 conjugated with IRDye800CW) in tumour-specific imaging to determine if it can help surgeons remove malignant lesions under fluorescence guidance.
\end{abstract}

Methods: The expression of OC183B2 antigen in epithelial ovarian cancer (EOC) tissues and cell lines was determined using immunohistochemistry (IHC). Western blotting was used to verify the expression of OC183B2 in SKOV3-Luc tumours. Antibodies against OC183B2 and mouse immunoglobulin G1 (IgG1) were conjugated with IRDye800CW to develop the antibody fluorescent probes COC183B2-800 and IgG-800 (immunoglobulin G1 conjugated with IRDye800CW). A subcutaneous mouse tumour model of SKOV3-Luc cells was constructed. Bioluminescent imaging (BLI) was conducted to detect the tumour location. Near-infrared fluorescence (NIRF) imaging was performed after the mice were injected with imaging agents. The mice were sacrificed $96 \mathrm{~h}$ postinjection, and the biodistribution assays were performed using NIRF imaging.

Results: In 69 EOC patients, the total positive rate of OC183B2 in EOC tissues was 89.9\% (62/69). Expression of the OC183B2 antigen was positive in SKOV3-LuC, 3AO, ES2 and A2780 cells. The OC183B2 antigen could be detected in SKOV3-Luc tumours. NIRF imaging of the COC183B2-800 probe at different doses showed a high fluorescent signal at the tumour location that was in line with the site detected by bioluminescent imaging. The tumour background ratio (TBR) was significantly higher in the COC183B2-800 group than in the lgG-800, IRDye800CW and PBS groups. The fluorescent probe COC183B2-800 is metabolized mainly through the liver and does not accumulate in other organs.

Conclusions: COC183B2-800 shows effective tumour-specific targeting of EOC and is a promising diagnostic and therapeutic tool for fluorescence-guided surgery.

Keywords: Ovarian cancer, Near-infrared fluorescence imaging, COC183B2-800, Surgery

\footnotetext{
*Correspondence: liyi@pkuph.edu.cn; cuiheng@pkuph.edu.cn

'Department of Obstetrics and Gynecology, Peking University People's

Hospital, No. 11, Xi-Zhi-Men South Street, Xi Cheng District, Beijing 100044,

China

Full list of author information is available at the end of the article
}

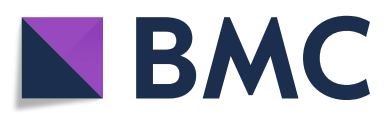

(๑) The Author(s). 2020 Open Access This article is licensed under a Creative Commons Attribution 4.0 International License, which permits use, sharing, adaptation, distribution and reproduction in any medium or format, as long as you give appropriate credit to the original author(s) and the source, provide a link to the Creative Commons licence, and indicate if changes were made. The images or other third party material in this article are included in the article's Creative Commons licence, unless indicated otherwise in a credit line to the material. If material is not included in the article's Creative Commons licence and your intended use is not permitted by statutory regulation or exceeds the permitted use, you will need to obtain permission directly from the copyright holder. To view a copy of this licence, visit http://creativecommons.org/licenses/by/4.0/. The Creative Commons Public Domain Dedication waiver (http://creativecommons.org/publicdomain/zero/1.0/) applies to the data made available in this article, unless otherwise stated in a credit line to the data. 


\section{Introduction}

Ovarian cancer is the most lethal gynaecological malignancy. Most patients are diagnosed with advanced ovarian cancer, and early detection of ovarian cancer is difficult due to nonspecific symptoms or a lack of symptoms [1]. Surgery is one of the most frequent and effective treatments for cancer and is often the first and only curative option [2]. Intraoperative visualization of tumours, including metastatic and microscopic lesions, is of significant importance for the outcome of therapy [3].

Conventional imaging techniques include magnetic resonance imaging (MRI), computed tomography (CT), $\mathrm{X}$-ray and ultrasound. However, the signal specificity and sensitivity of these imaging techniques are limited. Moreover, these imaging techniques cannot be conducted during surgery. Currently, surgeons mainly depend on visual inspection and palpation to identify lesions. The presence of occult lesions in the tumour margin and nonenlarged yet metastatic lymph nodes needs to be considered to determine how radical surgery should be. To detect these tiny lesions accurately during the operation, a highly sensitive intraoperative imaging modality is urgently needed. Near-infrared fluorescence (NIRF) imaging makes visualization of cancer a reality during the operation, and it could help realize complete resection of the tumour [2]. Furthermore, fluorescence imaging is safe and noninvasive with the advantages of high specificity and superior resolution.

We previously developed the monoclonal antibody COC183B2, which has high-affinity binding to epithelial ovarian cancer (EOC) tissue. It was produced using hybridoma technology in our laboratory. The antigen of COC183B2 is ovarian cancer-associated antigen OC183B2 [4, 5]. Our previous work showed that ${ }^{131} \mathrm{I}$ COC183B2 can localize EOC by radioimmunoimaging [6]. IRDye800CW is a NIRF dye that is cleared via the kidneys, and the extinction coefficient of IRDye800CW is high [7]. More importantly, IRDye800CW has already been extensively tested in clinical studies and proved safe in humans $[8,9]$. We conjugated COC183B2 with IRDye800CW and synthesized COC183B2-800. The imaging agent of COC183B2-800 needs to bind specifically to antigen OC183B2 in the tumour, facilitating specific imaging.

In our study, we developed and validated a novel EOC antigen-targeting fluorescent probe, COC183B2-800, for tumour-specific imaging of EOC that has high clinical translational potential.

\section{Materials and methods}

Immunohistochemistry (IHC) staining of EOC tissues

IHC staining was conducted in the human EOC tissues of 69 patients using COC183B2 antibody as previously described [10]. Mouse immunoglobulin G1 (IgG1) (Cat\# ab81032, Abcam, USA) was used as the negative control.

\section{Cell lines}

A total of four EOC cell lines (SKOV3-Luc, 3AO, ES2 and A2780) were used for OC183B2 antigen detection and other cell-based experiments. The 3AO, ES2 and A2780 cell lines were preserved in the Center of Gynecologic Oncology, Peking University People's Hospital. The SKOV3-Luc cell line expressing the firefly luciferase gene was kindly provided by Professor Xipeng Wang from Shanghai Jiao Tong University. SKOV3-Luc, 3AO, ES2 and A2780 cells were cultured in RPMI 1640 medium supplemented with $10 \%$ FBS and $1 \%$ penicillin and streptomycin.

\section{IHC staining of EOC cell lines}

Four vitro cultured cell lines (SKOV3-Luc, 3AO, ES2, A2780) representing different histological types of EOC were fixed using $4 \%$ formalin after harvest. The fixed cells were then mixed with melted agarose to create a block of dispersed single cells. The resulting cell gels were subjected to standard histoprocessing and paraffin embedding. IHC staining of paraffin sections of EOC cell lines (SKOV3-Luc, 3AO, ES2, A2780) was conducted in the same way as that of EOC tissues as previously described.

\section{Western blot detection of SKOV3-Luc tumour samples}

SKOV3-Luc tumours were excised from sacrificed mice with subcutaneously transplanted (the construction of a subcutaneous mouse tumour model of SKOV3-Luc cells is described below). Protein was extracted using RIPA buffer (Cell Signaling Technology, USA) on ice. Protein $(40 \mu \mathrm{g})$ was subjected to $10 \%$ sodium dodecyl sulfatepolyacrylamide gel electrophoresis (SDS-PAGE). Then, protein was transferred to a $0.45 \mu \mathrm{m}$ polyvinylidene fluoride membrane and incubated in blocking buffer $(5 \%$ fat-free milk powder dissolved in phosphate-buffered saline $[\mathrm{PBS}]$ ) for $1 \mathrm{~h}$ at room temperature. Membranes were incubated with primary antibodies against OC183B2 $(0.2 \mu \mathrm{g} / \mathrm{mL})$ and IgG1 isotype control $(0.2 \mu \mathrm{g} /$ $\mathrm{mL}$, Abcam, USA) overnight at $4{ }^{\circ} \mathrm{C}$. Membranes were imaged using an Odyssey CLx Imaging System (LI-COR Biotechnology) after incubation with IRDye800CW goat anti-mouse secondary antibody (diluted 1:10000, LICOR Biotechnology, USA) for $1 \mathrm{~h}$ at room temperature.

\section{Development of a tumour-specific fluorescent probe}

Antibodies against OC183B2 and IgG1 (Cat\# ab81032, Abcam, USA) were dissolved in PBS. The concentration of antibodies was $1 \mathrm{mg} / \mathrm{mL}$. The $\mathrm{pH}$ of the antibodies was raised to 8.5 by adding $1 \mathrm{M}$ sodium bicarbonate buffer. IRDye800CW (Cat\# 929-70021, LI-COR, USA) and 
antibodies were mixed (mole ratio $=1: 20$ ) and incubated overnight at $4{ }^{\circ} \mathrm{C}$ in the dark. The reaction product was purified using a Pierce Zeba desalting spin column (Cat\# 87766, Thermo Fisher Scientific, USA). The dye-protein ratio was measured using a UV-Vis system after purification of the IRDye800CW-conjugated COC183B2 antibody (COC183B2-800) and IgG1 (IgG-800).

\section{Subcutaneous mouse model of EOC and NIRF imaging}

The Medical Ethics Committee of Peking University People's Hospital approved the study (no. 2016PHC078). Five-week-old female BALB/c nude mice were injected with $5 \times 10^{6}$ SKOV3-luc cells in the right shoulder. Mice bearing 0.4-0.7 cm SKOV3-Luc tumours were injected with imaging agents. A total of 24 mice were used and randomly divided into 8 groups with 3 mice per group. Each group was injected with one of the following agents: (1) $50 \mu \mathrm{g}$ COC183B2-800, (2) $25 \mu \mathrm{g}$ COC183B2800, (3) $12.5 \mu \mathrm{g}$ COC183B2-800, (4) $50 \mu \mathrm{g}$ IgG-800, (5) $25 \mu \mathrm{g}$ IgG-800, (6) $12.5 \mu \mathrm{g}$ IgG-800, (7) IRDye800CW alone, or (8) PBS. All agents were dissolved in $100 \mu \mathrm{l}$ PBS and administered to mice via tail-vein injection.

To avoid fluorescent signal interference, NIRF imaging was conducted before bioluminescent imaging (BLI). From 6 to $96 \mathrm{~h}$ postinjection, all mice were fluorescently imaged at $800 \mathrm{~nm}$ to detect IRDye800CW using an IVIS Spectrum Imaging System. BLI was performed to localize SKOV3-Luc tumours. Briefly, $150 \mathrm{mg} / \mathrm{kg}$ D-luciferin was injected intraperitoneally $10 \mathrm{~min}$ before BLI. The tumour background ratio (TBR) was analysed by measuring the fluorescent signal of the tumour and that of normal tissue that exists around the tumour.

\section{Ex vivo imaging, biodistribution and histological verification}

All animals were sacrificed $96 \mathrm{~h}$ after injection of the agents. NIRF imaging of the excised organs was performed using an IVIS Spectrum Imaging System for biodistribution studies. Histology paraffin-embedded SKOV3-Luc tumour tissues were sectioned and stained with haematoxylin-eosin (H\&E). To confirm the presence of OC183B2, other sections were subjected to IHC staining using a COC183B2 antibody.

\section{Statistical analysis}

All fluorescent signal values and TBRs of different groups and time points are depicted as the mean \pm SD. Means of fluorescent signal values and TBRs were compared using one-way ANOVA or independent samples $t$ test. $P$ values lower than 0.05 were considered to indicate a significant difference. GraphPad Prism 5.0 (version 5.01, IBM Corp.) was used to generate graphs. SPSS 22.0 (IBM technologies) was used to perform statistical analysis.

\section{Results}

OC183B2 expression in EOC cell lines and human tissues

IHC staining was conducted in the EOC tissues of 69 patients. The total positive rate of OC183B2 expression was $89.9 \%(62 / 69)$. We also analysed the positive rate of OC183B2 expression in samples of different histologic types, different clinical stages and different pathological grades. The expression of OC183B2 was positive in 92.2\% (47/51) of serous EOCs, $25 \%$ (1/4) of mucinous EOCs, $100 \%(8 / 8)$ of clear cell EOCs and $100 \%(6 / 6)$ of endometrioid EOCs (Fig. 1A). There were 83.3\% (15/18) of stage I, $100 \%(6 / 6)$ of stage II, $92.9 \%(39 / 42)$ of stage III and $66.7 \%(2 / 3)$ of stage IV EOC patients expressing OC183B2 (Fig. 1B). The positive rates of OC183B2 in different pathological grades were 50\% $(3 / 6)$ in grade $1,100 \%(5 / 5)$ in grade 2 and $93.1 \%$ $(54 / 58)$ in grade 3 (Fig. 1C).

The result of IHC staining of cell lines representing different cell types showed that OC183B2 antigen expression was positive in SKOV3-Luc (serous EOC cell), $3 \mathrm{AO}$ (mucinous EOC cell), ES2 (clear cell EOC cell) and A2780 (endometrioid EOC cell) cells, which is consistent with the results of IHC staining of human EOC tissues (Fig. 2a).

\section{Western blot detection of SKOV3-Luc tumour samples}

Western blot experiments indicated that OC183B2 was expressed in SKOV3-Luc tumours. The isotype control IgG1 was negative in SKOV3-Luc tumours. The molecular weight of the OC183B2 antigen in SKOV3-Luc tumours was $56 \mathrm{kDa}$ and $25 \mathrm{kDa}$ (Fig. 2b), which is consistent with the results of our previous work [5].

\section{Development of a tumour-specific fluorescent probe}

After purification of the reaction product, antibodies conjugated with IRDye800CW were added into 96-well plates and imaged using an Odyssey CLx Imaging System. The results showed that the fluorescent signal could be detected with COC183B2-800, IgG-800 and IRDye800CW (Fig. 3c). The antibodies were conjugated with IRDye800CW successfully. The UV-Vis system confirmed that the dye-to-protein ratio of antibodies was 2.2:1.

\section{Specificity of COC183B2-800 in NIRF imaging in a} subcutaneous mouse tumour model

BLI was performed in all the mouse groups. NIRF imaging of the COC183B2-800 probe showed a high fluorescent signal in the tumour location, which is in line with the site found by BLI (Fig. 4). The IgG-800, IRDye800CW and PBS groups did not show tumour-specific signals (Fig. 4). The group of mice injected with $50 \mu \mathrm{g}$ COC183B2-800 agent had a significantly $(P<0.0001)$ higher TBR than the groups injected with $50 \mu \mathrm{g}$ IgG-800, IRDye800CW alone 


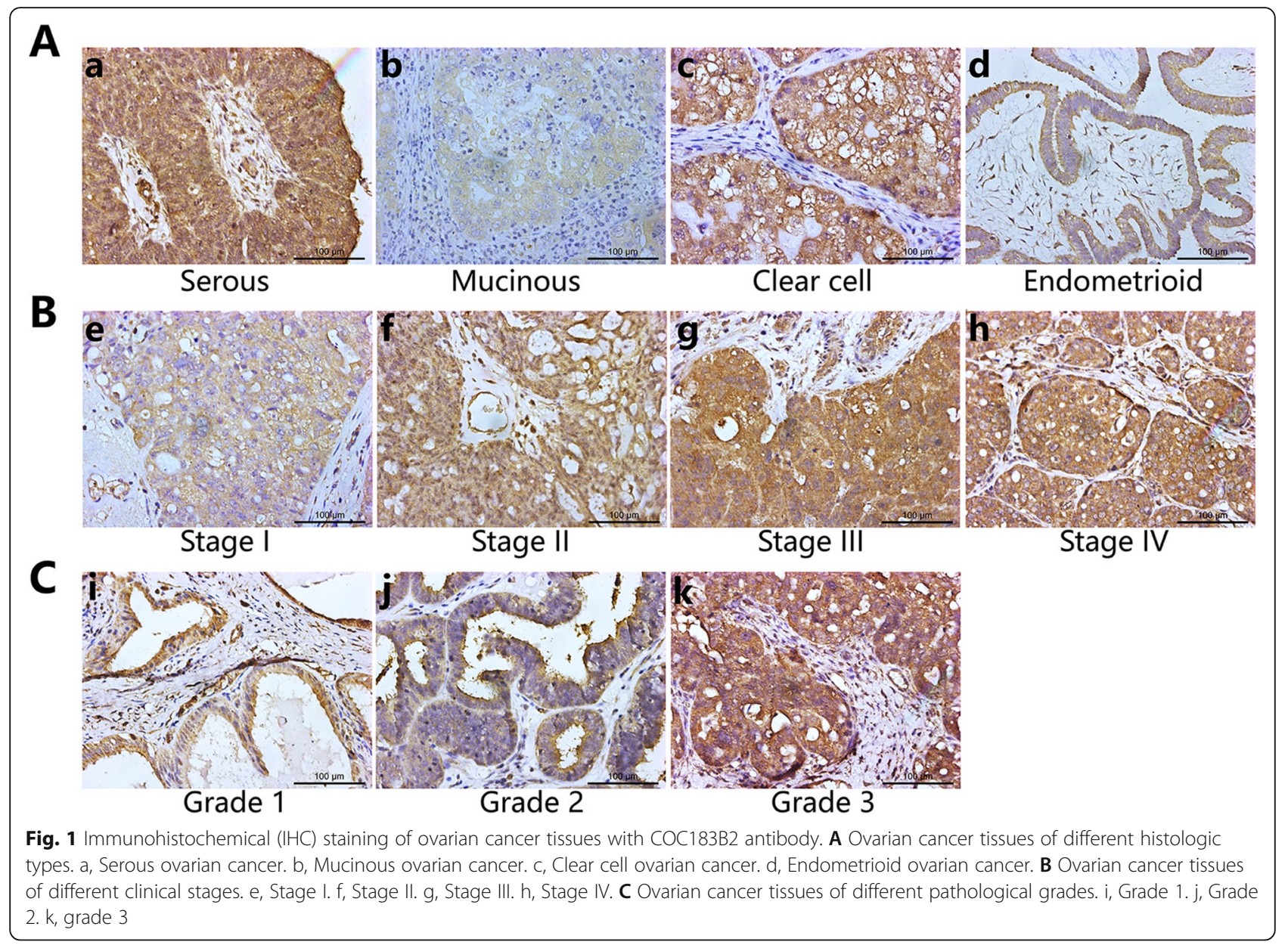

or PBS at $6 \mathrm{~h}(P<0.0001), 24 \mathrm{~h}(P<0.0001), 48 \mathrm{~h}(P<$ $0.0001), 72 \mathrm{~h}(P<0.0001)$ and $96 \mathrm{~h}(P<0.0001)$ postinjection (Fig. 5a). The TBR of mice injected with $25 \mu \mathrm{g}$ COC183B2-800 was significantly higher than that of mice injected with $25 \mu \mathrm{g}$ IgG-800, IRDye800CW alone or PBS at $6 \mathrm{~h}(P<0.0001), 24 \mathrm{~h}(P<0.0001), 48 \mathrm{~h}(P<0.0001)$, $72 \mathrm{~h}(P<0.0001)$ and $96 \mathrm{~h}(P<0.0001)$ after injection
(Fig. 5b). There was a significantly higher TBR in the $12.5 \mu \mathrm{g}$ COC183B2-800 group than in the $12.5 \mu \mathrm{g}$ IgG800 , IRDye800CW alone or PBS groups at $6 \mathrm{~h}(P<$ $0.0001), 24 \mathrm{~h}(P<0.0001), 48 \mathrm{~h}(P<0.0001)$ and $72 \mathrm{~h}(P$ $=0.002$ ) after injection (Fig. $5 \mathrm{c}$ ). There was increase in TBR as the dose of COC183B2-800 increased. High fluorescent signals were mainly concentrated in the
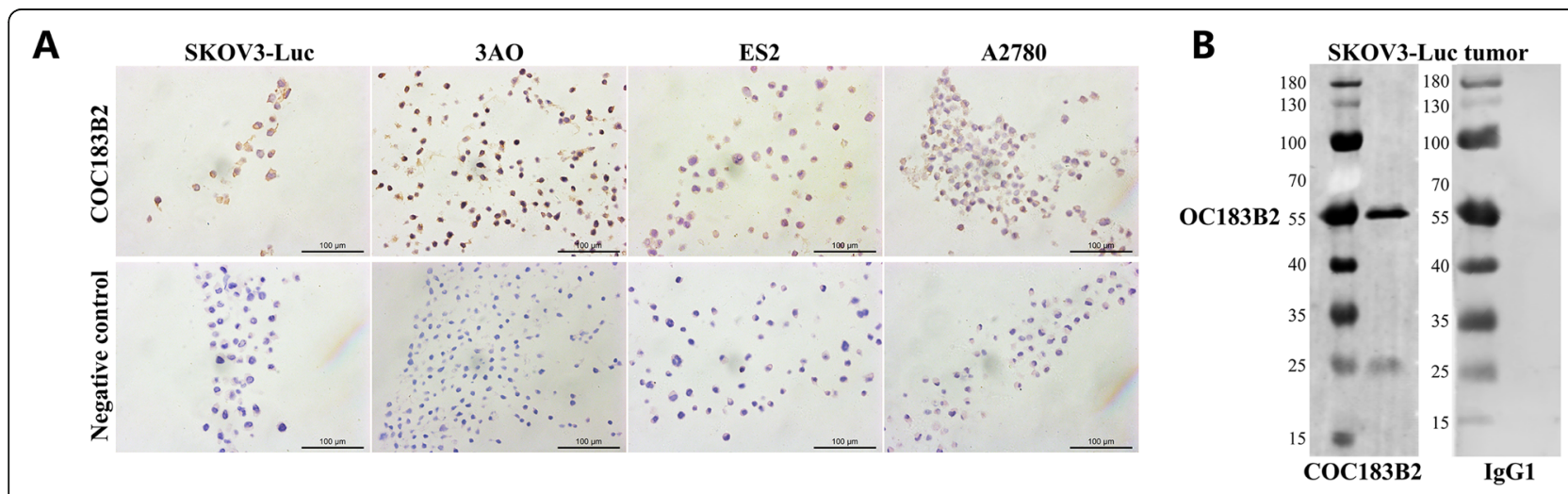

Fig. 2 The expression of OC183B2 in ovarian cancer cell lines and SKOV3-LuC tumour samples. a Immunohistochemical (IHC) staining of ovarian cancer cell lines (SKOV3-Luc, 3AO, ES2, A2780). b Western blot analysis of OC183B2 in SKOV3-Luc tumour samples 

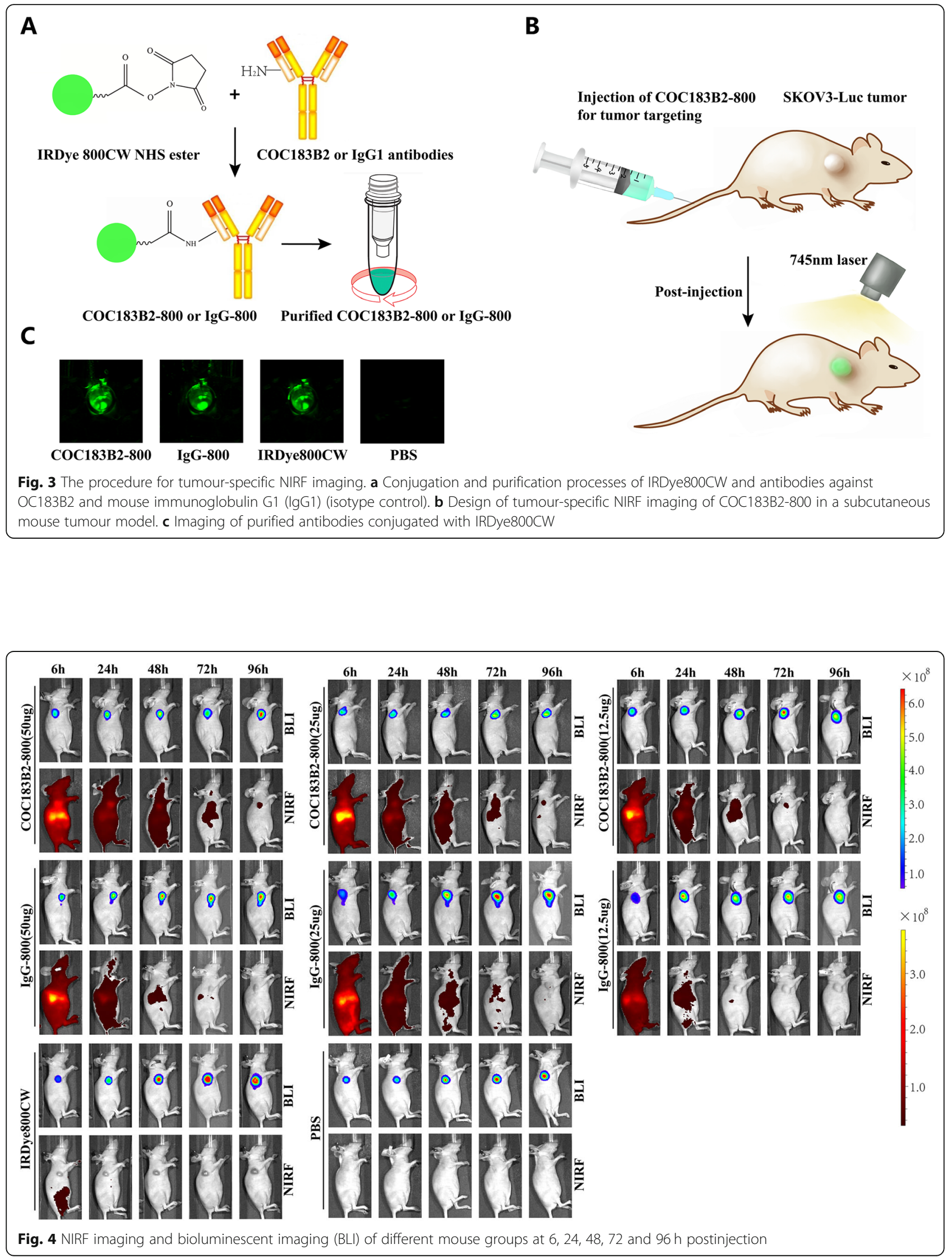


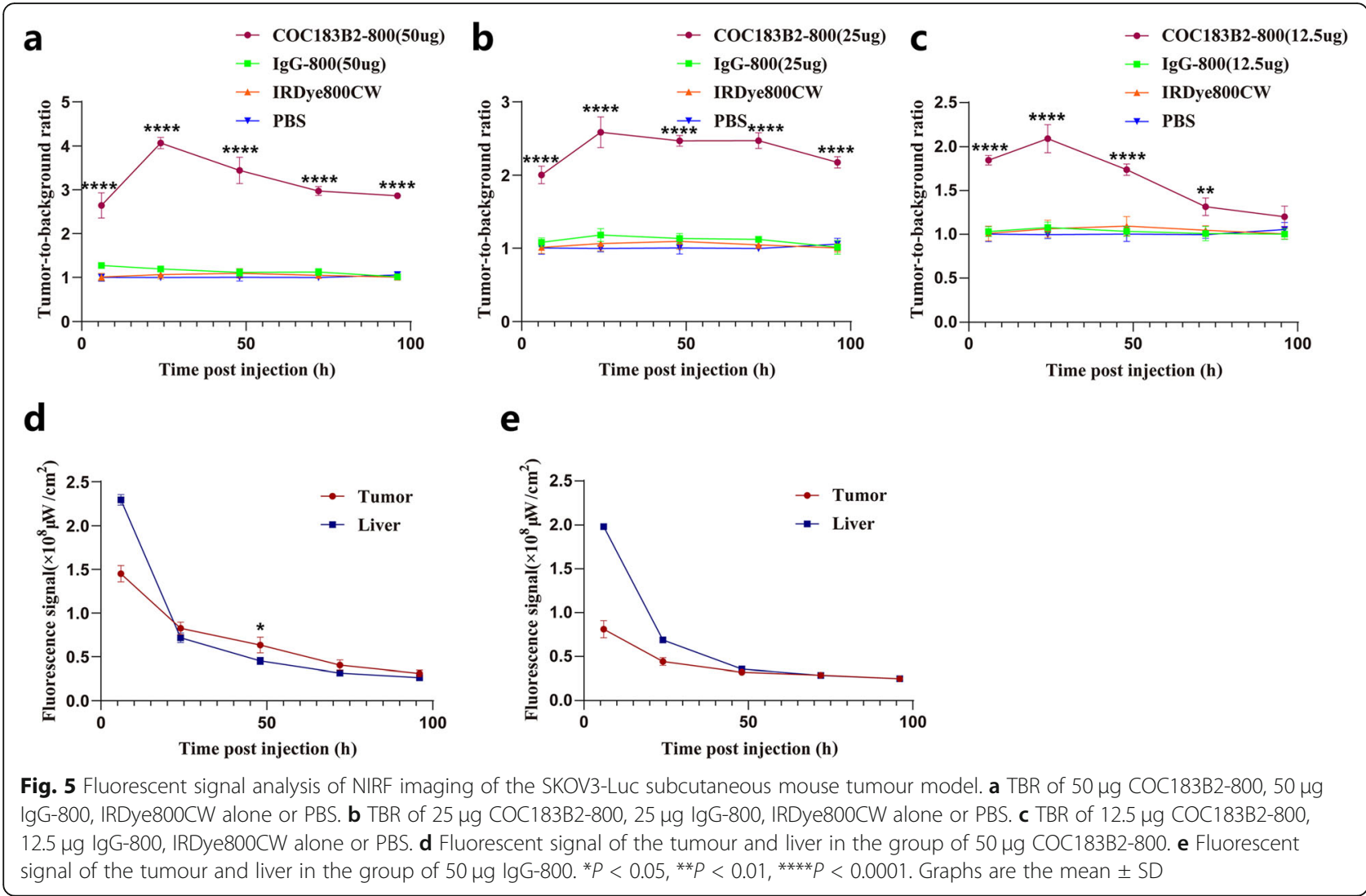

liver, and we compared the fluorescent signals of the liver and tumour in the COC183B2-800 and IgG-800 groups. The fluorescent signal of the tumour was significantly higher than that of the liver in the $50 \mu \mathrm{g}$ COC183B2-800 group at $48 \mathrm{~h}$ postinjection $(P=$ 0.0361; Fig. 5d).

\section{Ex vivo imaging, biodistribution and histological verification}

After the mice were sacrificed, the organs were excised. The results of $H \& E$ staining verified the excised tumour tissue. IHC staining of OC183B2 was positive in SKOV3-Luc tumours (Fig. 6b). Ex vivo NIRF imaging showed that there was still a strong fluorescent signal in the tumours of the $50 \mu \mathrm{g}$ COC183B2-800 group (Fig. $6 a)$. In the other groups, a strong fluorescent signal could only be detected in the liver (Fig. 6a). In the PBS control group, the lowest fluorescent signal that could be detected is $1.5 \times 10^{7} \mu \mathrm{W} / \mathrm{cm}^{2}$. Biodistribution assays of the $50 \mu \mathrm{g}$ COC183B2-800 group showed that the highest fluorescent signal was in the liver, and the second-highest fluorescent signal was in the tumour (Fig. 6c). The fluorescent signal was slightly higher in the kidney than in the rest of the organs. Relatively low fluorescent signals could be detected in other organs (heart, lung, spleen, ovary, uterus, muscle, pancreas, stomach and intestine) (Fig. 6c). In the IgG-800 group, the fluorescent signal in the liver was low, which may be caused by the shorter fluorescence lifetime of the nonspecific probe of IgG-800 compared with that of the specific antibody probe [11].

\section{Discussion}

To improve the survival of EOC patients, it is crucial to reduce the presence residual lesions after surgery. Currently, surgeons mainly depend on visual inspection and palpation to identify lesions. Conventional imaging modalities, such as MRI, CT and PET/CT, have a limitation of low sensitivity and specificity. Subcentimetre lesions are usually difficult to identify by CT and MRI. PET/CT is usually performed to determine the site of metastasis before surgery for recurrent cancer. However, PET/CT is not sensitive enough to detect clear cell or mucinous carcinoma, tumours with postchemotherapy metabolic inhibition, cystic lesions and tiny lesions, which are factors that affect surgical decisions. Our immunohistochemical experiments showed that clear cell carcinomas expressed OC183B2. Theoretically, COC183B2-800 probes can identify such tumours. Moreover, conventional imaging modalities cannot be performed intraoperatively or guide surgery by imaging. Surgeons may miss subcentimetre lesions and metastatic lesions [12]. 


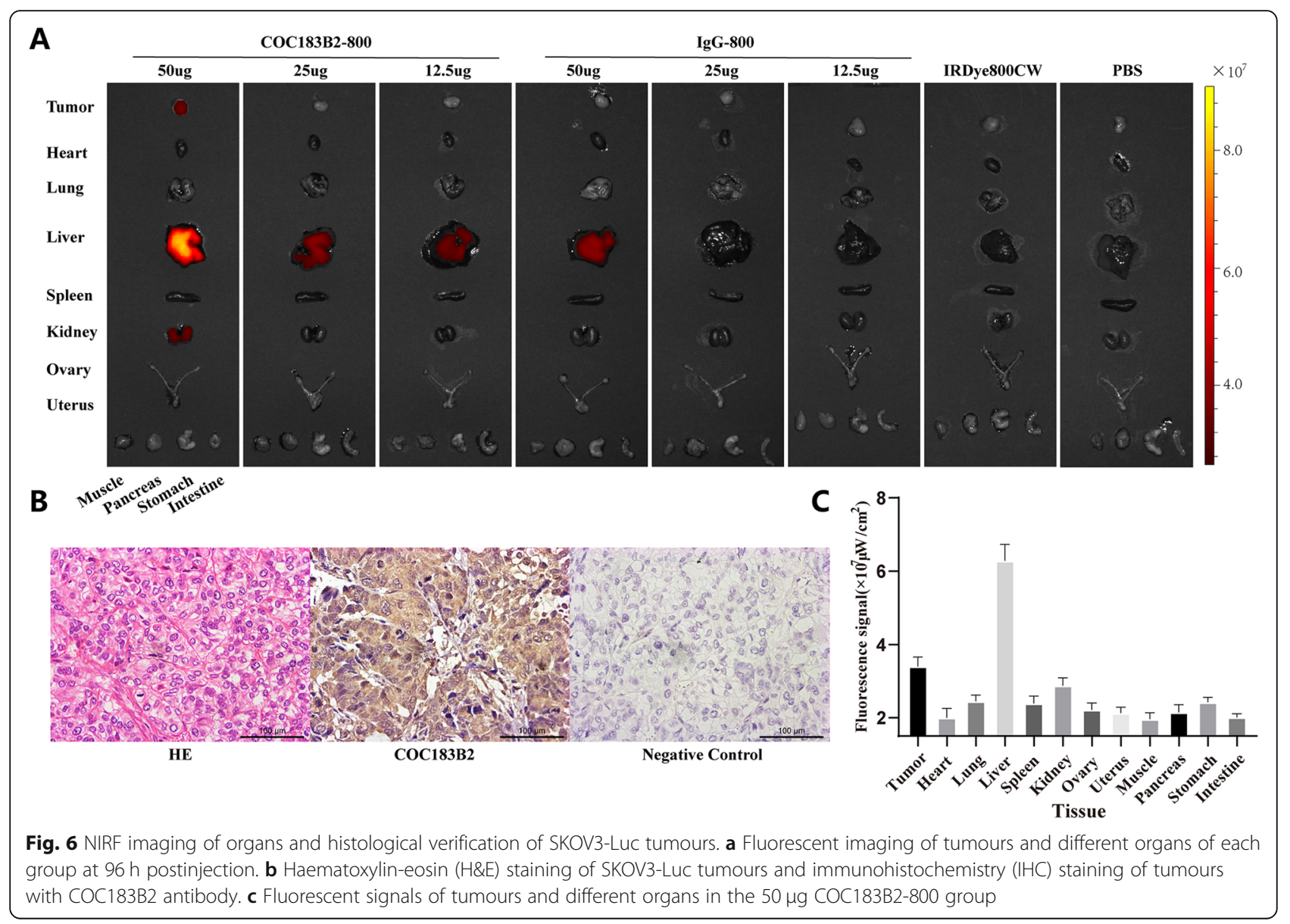

To overcome the limitations of conventional imaging modalities and guide surgery, highly specific intraoperative in vivo fluorescent imaging that has the advantages of high specificity, superior resolution and real-time detection is needed. Tumour-specific fluorescence-guided surgery could guide the resection of tumour-positive margins, tiny lesions and tumour-positive lymph nodes and improve survival by reducing the presence of residual disease. Previously, Hutteman et al. used indocyanine green (ICG), a nontargeted optical imaging agent, to detect pancreatic ductal adenocarcinoma. However, after intravenous injection of ICG, no useful tumour demarcation could be visualized [13]. ICG lacks imaging specificity, which will lead to the misdiagnosis of positive lesions. Therefore, targeted optical imaging, which depends on the binding of antibodies to antigens or ligands to receptors, is needed to replace nontargeted optical imaging. Clinical trials of targeted tumourspecific fluorescence imaging conducted in different malignancies have shown remarkable success [14-17]. Though ICG can be used in humans, it lacks active groups to conjugate with ligands or antibodies. Most tumour-specific fluorescent probes use IRDye800CW since IRDye800CW has already been extensively tested in clinical studies and can be conjugated to ligands or antibodies [18].
In previous studies, the tumour targets of ovarian cancer-specific fluorescence imaging included folate receptor alpha (FR- $\alpha)[19,20]$, follicle-stimulating hormone receptor (FSHR) [2], anti-human epidermal growth factor receptor 2 (HER2) [21] and so on. However, the widespread expression of HER2 in normal human tissues impacts the specificity and accuracy of imaging [22]. The relatively low expression rate (56.4\%) of FSHR may impact the universality of imaging [23]. In a clinical trial of fluorescence imaging based on the target FR- $\alpha$, false-positive fluorescence was identified in 17/ 50 nonmetastatic lymph nodes caused by OTL-38 targeting of FR $\beta$, which is expressed by tumour-associated activated macrophages [24]. Therefore, to better improve ovarian cancer-specific fluorescence-guided surgery, it is necessary to seek and develop better tumour targets or targets that could be functionally complementary to known targets. We previously developed the monoclonal antibody COC183B2, which has high-affinity binding to EOC tissue. IHC staining was conducted in the human EOC tissues of 69 patients. The total positive rate of OC183B2 expression was $89.9 \%(62 / 69)$. The expression of OC183B2 could be detected in different histologic types, different clinical stages and different pathological 
grades of EOC. Therefore, the COC183B2-800 fluorescence probe has extensive applicability in a wide range of EOC patients. Furthermore, the OC183B2 antigen is rarely expressed in normal tissues, guaranteeing the specificity of imaging [6]. Our previous study of radioimmunoimaging via ${ }^{131} \mathrm{I}-\mathrm{COC} 183 \mathrm{~B} 2$ in patients suggests that the COC183B2 antibody can be used for radioimmunoimaging to identify the location of ovarian cancer [6].

However, both imaging probes and the equipment for imaging of ${ }^{131} \mathrm{I}$-COC183B2 have a radiation risk. Moreover, the lesions of patients are not visible during the operation due to the imaging modality. We later conjugated ultrasmall superparamagnetic iron oxide nanoparticles (USPIOs) and Cy7 with COC183B2 antibody and achieved early detection of EOC through OC183B2targeted MRI and fluorescence imaging [10, 25]. However, antibodies conjugated with USPIOs might be toxic. Cy7 has not been approved for use in humans, and our previous work showed the phenomenon of lung accumulation of Cy7-conjugated COC183B2 [10]. As previously described, IRDye800CW is a safe and effective NIRF dye. Therefore, we conjugated COC183B2 to IRDye800CW and synthesized COC183B2-800. The results of IHC staining of cell lines representing different cell types showed that the OC183B2 antigen expression was positive in SKOV3-Luc (serous EOC cell), 3AO (mucinous EOC cell), ES2 (clear cell EOC cell) and A2780 (endometrioid EOC cell) cells, which is consistent with the result of IHC staining of human EOC tissues. SKOV3-Luc cells represent serous EOC which accounts for approximately $70 \%$ of all EOCs. IHC staining intensity of different cell types showed that the expression of OC183B2 in SKOV3-Luc cells was relatively higher, and the western blot results also showed that OC183B2 was expressed in SKOV3-Luc tumours. Thus, the SKOV3Luc cell line was suitable for further study in a subcutaneous mouse model.

The NIRF imaging of the COC183B2-800 probe showed a high fluorescent signal at the tumour location, which was in line with the site detected by BLI. COC183B2-800 showed excellent tumour-specific targeting in the subcutaneous mouse tumour model. At different time points $(6,24,48,72$ and $96 \mathrm{~h}$ postinjection), mice in the groups with $50 \mu \mathrm{g}$ and $25 \mu \mathrm{g}$ COC183B2-800 all showed significantly higher TBR than the corresponding groups of IgG-800, IRDye $800 \mathrm{CW}$ and PBS. In the $12.5 \mu \mathrm{g}$ COC183B2-800 group, TBR was significantly higher at 6, 24, 48 and $72 \mathrm{~h}$ than it was in the other groups, but this effect was not observed at $96 \mathrm{~h}$. The TBR declined as the dose of COC183B2-800 was reduced. The proper dose of COC183B2-800 for imaging may be $25 \sim 50 \mu \mathrm{g}$. According to the change in TBR over time, 24 72 $\mathrm{h}$ postinjection may be the best period of time for imaging. Furthermore, the fluorescent probe COC183B2-
800 was metabolized mainly through the liver and did not accumulate in other organs. COC183B2-800 is a very safe and effective ovarian cancer-specific fluorescent probe.

\section{Conclusions}

In conclusion, we developed the novel ovarian cancerspecific antibody fluorescent probe COC183B2-800. Through the evaluation of COC183B2-800 in a subcutaneous mouse model of EOC, we found that COC183B2800 could bind selectively to the EOC antigen OC183B2. COC183B2-800 exhibited excellent TBR, and there was a clear correlation between the histopathologic evidence and the fluorescent signal. Ovarian cancer-specific imaging was achieved, with favourable liver and kidney clearance. IRDye800CW has already been proven safe to be used in humans, and our experiment paves the way for clinical translation of the fluorescence imaging agent COC183B2-800.

\section{Abbreviations}

EOC: Epithelial ovarian cancer; IHC: Immunohistochemistry;

IgG1: Immunoglobulin G1; NIRF: Near-infrared fluorescence;

BLI: Bioluminescent imaging; TBR: Tumour background ratio; MRI: Magnetic resonance imaging; CT: Computed tomography

\section{Acknowledgements}

The authors are very grateful to Professor Xipeng Wang from Shanghai Jiao Tong University for kindly providing SKOV3-Luc cells.

\section{Authors' contributions}

$\mathrm{HC}$ and $\mathrm{YL}$ contributed to the research design and manuscript writing. JCC carried out the main experiments, performed statistical analysis and drafted the manuscript. CZ provided some advice. YXG carried out some of the experiments. RQM and XHC provided technical guidance. XY carried out some of the experiments. HYC provided experimental guidance. All authors read and approved the final manuscript.

\section{Funding}

This work was financially supported by the National Key Research and Development Program of China (no. 2016YFA0201404)

\section{Availability of data and materials}

Not applicable.

\section{Ethics approval and consent to participate}

The Medical Ethics Committee of Peking University People's Hospital approved the study (no. 2016PHC078).

\section{Consent for publication}

Not applicable.

\section{Competing interests}

The authors declare that they have no competing interests.

\section{Author details}

${ }^{1}$ Department of Obstetrics and Gynecology, Peking University People's Hospital, No. 11, Xi-Zhi-Men South Street, Xi Cheng District, Beijing 100044, China. ${ }^{2}$ Center of Gynecologic Oncology, Peking University People's Hospital, No. 11, Xi-Zhi-Men South Street, Xi Cheng District, Beijing 100044, China.

Received: 8 February 2020 Accepted: 25 March 2020

Published online: 06 April 2020

References

1. Jelovac $D$, Armstrong DK. Recent progress in the diagnosis and treatment of ovarian cancer. CA-Cancer J Clin. 2011;61:183-203. 
2. Wang P, Fan Y, Lu L, Liu L, Fan L, Zhao M, et al. NIR-II nanoprobes in-vivo assembly to improve image-guided surgery for metastatic ovarian cancer. Nat Commun. 2018;9:1-10.

3. Vahrmeijer $A L$, Hutteman $M$, van der Vorst JR, van de Velde $\mathrm{CJH}$, Frangioni $J V$. Image-guided cancer surgery using near-infrared fluorescence. Nat Rev Clin Oncol. 2013;10:507-18.

4. Qian HN, Feng J, Cui H, Fu TY, Wei P, Fu ZY. Generation and characterization of three monoclonal antibodies to human ovarian epithelial adenocarcinomas. Chinese Med J-Peking. 1989;102:839-43.

5. Guo HF, Feng J, Zhang H, Yao Y, Cheng HY. Purification and characterization of corresponding antigen recognized by monoclonal antibody $183 \mathrm{~B} 2$ against ovarian carcinoma. Zhonghua fu chan ke za zhi. 2005:40:614-8

6. Qian HN, Feng J, Cui H, Gao BS, Qin SL, Fu TY, et al. A preliminary study of radioimmunoimaging by $1311-C O C 183 B 2$ monoclonal antibody in patients with epithelial ovarian cancer. Chinese Med J-Peking. 1991;104:109-13.

7. Tummers WS, Kimura RH, Abou-Elkacem L, Beinat C, Vahrmeijer AL, Swijnenburg RJ, et al. Development and preclinical validation of a cysteine knottin peptide targeting integrin alphavbeta6 for near-infrared fluorescentguided surgery in pancreatic cancer. Clin Cancer Res. 2018;24:1667-76.

8. Rosenthal EL, Warram JM, de Boer E, Chung TK, Korb ML, Brandwein-Gensler $M$, et al. Safety and tumor specificity of cetuximab-IRDye800 for surgical navigation in head and neck cancer. Clin Cancer Res. 2015;21:3658-66.

9. Lamberts LE, Koch M, de Jong JS, Adams ALL, Glatz J, Kranendonk MEG, et al. Tumor-specific uptake of fluorescent bevacizumab-IRDye800CW microdosing in patients with primary breast cancer: a phase I feasibility study. Clin Cancer Res. 2017;23:2730-41.

10. Zhang C, Ling X, Guo Y, Yuan C, Cheng H, Ye X, et al. Evaluation of COC183B2 antibody targeting ovarian cancer by near-infrared fluorescence imaging. Chin J Cancer Res. 2019;31:673-85.

11. Pal R, Kang H, Choi HS, Kumar ATN. Fluorescence lifetime-based tumor contrast enhancement using an EGFR antibody-labeled near-infrared fluorophore. Clin Cancer Res. 2019;25:6653-61.

12. Lee YJ, Lee JM, Lee JS, Lee HY, Park BH, Kim YH, et al. Hepatocellular carcinoma: diagnostic performance of multidetector CT and MR imaging-a systematic review and meta-analysis. Radiology. 2015;275:97-109.

13. Hutteman M, van der Vorst JR, Mieog JSD, Bonsing BA, Hartgrink HH, Kuppen PJK, et al. Near-infrared fluorescence imaging in patients undergoing pancreaticoduodenectomy. Eur Surg Res. 2011;47:90-7.

14. van Keulen S, Nishio N, Fakurnejad S, Birkeland A, Martin BA, Lu G, Zhou O et al. The clinical application of fluorescence-guided surgery in head and neck cancer. J Nucl Med. 2019;60:758-63.

15. van Dam GM, Themelis G, Crane LM, Harlaar NJ, Pleijhuis RG, Kelder W, et al. Intraoperative tumor-specific fluorescence imaging in ovarian cancer by folate receptor-alpha targeting: first in-human results. Nat Med. 2011;17: 1315-9.

16. de Jongh SJ, Tjalma JJJ, Koller M, Linssen MD, Vonk J, Dobosz M, et al. Backtable fluorescence-guided imaging for circumferential resection margin evaluation in locally advanced rectal cancer patients using bevacizumab800CW. J Nucl Med. 2019;119.

17. Koller M, Qiu SQ, Linssen MD, Jansen L, Kelder W, de Vries J, et al. Implementation and benchmarking of a novel analytical framework to clinically evaluate tumor-specific fluorescent tracers. Nat Commun. 2018; 9:1-11.

18. Zhang RR, Schroeder AB, Grudzinski JJ, Rosenthal EL, Warram JM, Pinchuk $A N$, et al. Beyond the margins: real-time detection of cancer using targeted fluorophores. Nat Rev Clin Oncol. 2017;14:347-64.

19. Hekman MCH, Boerman OC, Bos DL, Massuger L, Weil S, Grasso L, et al. Improved intraoperative detection of ovarian cancer by folate receptor alpha targeted dual-modality imaging. Mol Pharm. 2017;14:3457-63.

20. Hoogstins CE, Tummers QR, Gaarenstroom KN, de Kroon CD, Trimbos JB, Bosse T, et al. A novel tumor-specific agent for intraoperative near-infrared fluorescence imaging: a translational study in healthy volunteers and patients with ovarian cancer. Clin Cancer Res. 2016;22:2929-38.

21. Terwisscha van Scheltinga AG, van Dam GM, Nagengast WB, Ntziachristos $V$, Hollema H, Herek JL, et al. Intraoperative near-infrared fluorescence tumor imaging with vascular endothelial growth factor and human epidermal growth factor receptor 2 targeting antibodies. J Nucl Med. 2011;52:1778-85.

22. Fagerberg L, Hallström BM, Oksvold P, Kampf C, Djureinovic D, Odeberg J, et al. Analysis of the human tissue-specific expression by genome-wide integration of transcriptomics and antibody-based proteomics. Mol Cell Proteomics. 2014;13:397-406.

23. Perales-Puchalt A, Svoronos N, Rutkowski MR, Allegrezza MJ, Tesone AJ, Payne KK, et al. Follicle-stimulating hormone receptor is expressed by most ovarian cancer subtypes and is a safe and effective immunotherapeutic target. Clin Cancer Res. 2017;23:441-53.

24. Boogerd LSF, Hoogstins CES, Gaarenstroom KN, de Kroon CD, Beltman JJ, Bosse T, et al. Folate receptor-a targeted near-infrared fluorescence imaging in high-risk endometrial cancer patients: a tissue microarray and clinical feasibility study. Oncotarget. 2018;9:791-801.

25. Quan G, Du X, Huo T, Li X, Wei Z, Cui H, et al. Targeted molecular imaging of antigen OC183B2 in ovarian cancers using MR molecular probes. Acad Rrdiol. 2010;17:1468-76.

\section{Publisher's Note}

Springer Nature remains neutral with regard to jurisdictional claims in published maps and institutional affiliations.
Ready to submit your research? Choose BMC and benefit from:

- fast, convenient online submission

- thorough peer review by experienced researchers in your field

- rapid publication on acceptance

- support for research data, including large and complex data types

- gold Open Access which fosters wider collaboration and increased citations

- maximum visibility for your research: over $100 \mathrm{M}$ website views per year

At BMC, research is always in progress.

Learn more biomedcentral.com/submissions 